\title{
"Yo soy mi cuerpo." La concepción henryana del ego como emergencia de una subjetividad corporal desde la inmanencia radical y patética de la Vida
}

\author{
"I am my body." The henryan conception of the ego as \\ emergence of a corporal subjectivity from the radical and \\ pathetic immanence of Life
}

VÍCTOR TEBA DE LA FUENTE*

\begin{abstract}
Resumen: En su radicalización de la fenomenología, Michel Henry (1922-2002) identifica la esencia del aparecer, condición de posibilidad de la manifestación de lo real, con la autorrevelación de la Vida en el espacio de la inmanencia radical y patética. Desde este esquema y siguiendo el pensamiento de Maine de Biran, Henry propone una concepción del "yo" como "cuerpo subjetivo", sentido y vivenciado como poder de acción y pasividad radical en la que se produce la autoafección de la Vida. Esta interpretación de la subjetividad plantea unas implicaciones espirituales, éticas y políticas que abren la reflexión filosófica a nuevos campos de investigación.

Palabras clave: Michel Henry, fenomenología radical-material, esencia del aparecer, cuerpo subjetivo, inmanencia patética, comunidad de vivientes
\end{abstract}

\begin{abstract}
In his radicalization of phenomenology, Michel Henry (1922-2002) identifies the essence of the appear, condition of the possibility of the reality's manifestation, with the self-revelation of Life in the space of radical and pathetic immanence. From this scheme and following the Maine de Biran's thought, Henry propose a conception of the "I" as "subjective body", felt and experienced as action power and radical passivity in which the auto-affection of Life happens. This interpretation of the subjectivity suggests some spiritual, ethical and political implications that bring the philosophical reflection to new research fields.

Keywords: Michel Henry, radical-material phenomenology, essence of the appear, subjective body, pathetic immanence, community of livings
\end{abstract}

\section{Introducción}

Es un hecho incuestionable que, si algo ha marcado las directrices de la filosofía moderna de forma realmente significativa, ha sido la apuesta cartesiana por el ego cogito como ver-

Fecha de recepción: 14/06/2016. Fecha de aceptación: 20/07/2016.

* Miembro del Personal Docente e Investigador en Formación (PDIF) del Departamento de Filosofía II de la Universidad de Granada (UGR). Intereses de investigación: Michel Henry, fenomenología francesa, filosofía de la religión, teoría queer y derechos LGTBI. Correo electrónico: vteba@ugr.es La presente comunicación se enmarca en el seno de actividades financiadas por las ayudas para la formación del profesorado universitario (Becas FPU) del Ministerio de Educación, Cultura y Deporte. 
dadera certeza autoevidente sobre la que fundamentar el conocimiento y la realidad. Y, con ella, también una concepción de la subjetividad como esa actividad pensante, con existencia independiente del cuerpo, de la cual es imposible dudar y que no es otra cosa sino "mi yo". La filosofía contemporánea, sin embargo, motivada por las investigaciones de la fenomenología y las aportaciones de corrientes como el enactivismo, empezó ya hace un tiempo a traer al campo de la reflexión filosófica el papel que la inserción, irremediablemente corporal, de la existencia humana en el mundo juega en la emergencia de la subjetividad. En la presente comunicación, se propone un análisis breve, pero no por ello menos profundo, de la reflexión en torno a la experiencia corporal que realiza Michel Henry, uno de esos autores realmente preocupados por la dimensión corporal del "yo", verdadero motor de su empresa filosófica y Leitmotiv de su obra, ya desde su primera tesis, Filosofía y fenomenología del cuerpo (trabajo publicado, no obstante, con posterioridad a su tesis de habilitación, La esencia de la manifestación).

Partiré, en primer lugar, de una presentación un tanto somera de las ideas que articulan el sentido general del proyecto filosófico henryano sirviéndome, para ello, no solo de sus principales obras, sino también de alguno de los capítulos introductorios que los estudiosos españoles de Henry ofrecen en las ediciones en castellano de sus libros y de alguno de los artículos que, sobre el pensamiento henryano, se han venido publicando. A continuación, intentaré mostrar la distinción entre los "tres cuerpos" de los que nos habla Henry siguiendo a Maine de Biran (filósofo contemporáneo de Descartes pero crítico con la antropología intelectualista cartesiana), para clarificar, en la medida de lo posible, su idea de la emergencia de la subjetividad como "resultado" de una autoafección de la Vida a sí misma por mediación del cuerpo propio. Finalmente, señalaré superficialmente algunas de las implicaciones de la fenomenología henryana del cuerpo en determinados ámbitos de nuestra realidad más cotidiana, utilizando la lectura que Michel Henry realiza de instancias existenciales vinculadas a nuestra dimensión corporal. La presente comunicación se perfila, así, como un acercamiento a la filosofía henryana del cuerpo que puede ser utilizada como estado de la cuestión para futuras investigaciones.

\section{Una fenomenología radical y material}

Michel Henry (1922-2002), filósofo y novelista francés todavía relativamente desconocido en España, entronca con la tradición fenomenológica (aunque de una manera heterodoxa o, sí se quiere, herética, como señalaré a continuación) porque recoge en sus obras los principios fundamentales de dicha tradición (en concreto, la identificación principal entre el ser de lo real y su aparecer), pero intenta al mismo tiempo, trascenderla. Como dice Tomás Domingo en su introducción a La barbarie (Domingo Moratalla, 2006, 8), lo que ocurre es que Henry "se mantiene dentro de la fenomenología, pero, como casi todos los fenomenólogos, quiere radicalizarla". Podemos esbozar, así, las notas principales de una fenomenología que el propio Henry describe como radical (porque busca la raíz del fenómeno, la raíz de lo que aparece -lo que no se manifiesta y, sin embargo, es la fuente de la manifestación-) y material (porque busca aquello de lo que está compuesto el aparecer, la sustancia de la que está hecha la manifestación, la condición material de posibilidad del fenómeno). Surge, pues, una fenomenología "arqueológica", que, recogiendo los postulados husserlianos y 
heideggerianos, intenta superarlos en busca de aquello que sostiene el aparecer y es su fundamento, su arché, su Comienzo, el origen material que, detrás de la apariencia, constituye el verdadero ser de los fenómenos (Cazzanelli, 2013, 5 y 6).

En este intento de radicalizar la fenomenología, Michel Henry se propone llevar a sus últimas consecuencias la "reducción fenomenológica" husserliana y la "diferencia ontológica" heideggeriana con el objeto de separar de manera definitiva el ser del ente, buscando más allá (en realidad, más acá) de la exterioridad del mundo, la instancia sobre la que descansaría la verdadera posibilidad del aparecer de la realidad. Para Michel Henry, la tradición de la "fenomenología histórica" de la que se quiere distanciar sigue presa de un prejuicio que ha acompañado a la filosofía a lo largo de su desarrollo histórico, a saber, la necesidad de presentar el fenómeno en la "distancia fenomenológica": a la luz de estas filosofías, lo que se nos presenta, se presenta como ya siempre separado, de forma que el ser se concibe en la exterioridad trascendental, en un ek-stasis, en una ruptura y separación originarias (Domingo Moratalla, 2006, 9).

En su análisis del parágrafo séptimo de Ser y tiempo, Henry señala que cuando Heidegger desarrolla su estudio etimológico del término "fenómeno", está manifestando este prejuicio de la trascendencia intramundana, propio de toda la tradición filosófica desde el pensamiento griego, según Henry. Lo que dice Heidegger es que el término "fenómeno" (phainomenon) deriva del verbo phainesthai, mostrarse; de forma que "fenómeno" significaría "lo que se muestra". Pero phainesthai deriva, a su vez, de la raíz pha-, que significa "luz", "claridad"; es decir, el ámbito en lo que algo puede hacerse visible. "Fenómeno" sería, pues, "mostrarse viniendo a la claridad" o "hacerse visible al salir a la luz". Así, el ente (lo que se muestra), el ser (lo que permite mostrarse) y la diferencia entre ambos, se conciben ya, desde la filosofía griega, según el paradigma de la luz; y la luz no es sino el horizonte del mundo: el fenómeno es lo que se muestra en la apertura del horizonte del mundo, lo que se ilumina en la claridad intramundana. Por eso dice Henry que la filosofía, desde el comienzo, está enferma o pervertida por esta exclusiva referencia a la trascendencia de la exterioridad; porque el concepto mismo de fenómeno ya es, desde el principio, un concepto mundano: aparecer es darse en el horizonte del mundo, venir a la luz del Afuera intramundano.

Para Henry, tanto Heidegger como Husserl se equivocan, pues confunden la esencia última del aparecer con su forma de darse en el espacio exterior de la conciencia (Husserl) o del tiempo (Heidegger). En esta investigación, Henry acabará considerando que la pureza de la materia originaria que es pervertida posteriormente por el pensamiento corresponde, en realidad, a la pura sensación, a lo que afecta: Henry quiere romper el prejuicio occidental de la coincidencia entre ser y pensamiento, presente en la tradición filosófica ya desde Parménides, concibiendo la manifestación originaria de la realidad como autoafección sustraída a toda exterioridad, pura materialidad incandescente que se manifiesta por sí y a sí misma en la inmanencia radical y sin mediación de ningún elemento que remita a la trascendencia de la exterioridad. Así, la recepción por la que llega al hombre todo lo que "se le aparece" no se concibe, en Henry, como el acto de recibir algo distinto de sí mismo desde una proveniencia "externa", sino como el acto (afección) o la capacidad permanente (afectividad) de recibirse a sí mismo, una "auto-recepción" que recibe el nombre de autoafección en la inmanencia radical (Domínguez Basalo, 1978, 168). 
La esencia de la conciencia no consiste, pues, ni en abrir un horizonte de sentido ni en recibirlo, sino en su poder para recibirse a sí misma, en este proceso de autoafección, un poder cuya esencia consiste en la identidad entre ser y aparecer. El trabajo filosófico de Henry puede resumirse, en palabras de Mario Lipsitz, como "la afirmación filosóficamente original del carácter absolutamente fenomenológico del ser como afectividad ontológica fundante cuyo modo de revelación es la inmanencia" (Lipsitz, 2000, 1). Y la inmanencia no es sino aquel espacio especialmente íntimo (en el sentido latino del término intimus, como superlativo de interior: "lo más adentro de"), donde no existe una distancia entre el puro sentir y un correlato objetivo del mismo. Todo esto no puede sino remitirnos a una reflexión en torno al cuerpo, porque ¿qué hay más inmanente a nosotros mismos, qué hay más íntimo que nuestro propio cuerpo? Pero entonces, ¿somos solo cuerpo?; ¿dónde queda la instancia que la tradición filosófica ha ido señalando como la psique, el alma, el espíritu, la res cogitans, la mente, etc.?

\section{El problema del alma. Hacia una subjetividad corporal}

El problema tradicional de la existencia del alma y su relación con el cuerpo es, para Henry, el problema de la subjetividad misma, el problema del ser del yo; un problema que, además, entronca con el sentido mismo de todo el "quehacer filosófico" desde que Heidegger señalara que se puede resolver el problema capital de la filosofía (la cuestión en torno al ser, la búsqueda de una respuesta a la pregunta “¿por qué el ser y no más bien la nada?”) resolviendo el problema yo; pues el hecho fundamental que define al hombre en su esencia propia es la comprensión del ser. Este problema del yo no es sino la imposibilidad del sujeto para alcanzarse realmente a sí mismo y reside en el hecho de que, según el prejuicio de la trascendencia intramundana que rechaza Henry, el sujeto sólo se puede conocer como objeto, pues incluso en el movimiento de la autorreflexión, la conciencia se "capta" intencionalmente a sí misma como "algo distinto de sí misma". Para Henry, no obstante, esto sólo ocurre si nos seguimos moviendo en el paradigma del conocimiento, puesto que más allá de él (en realidad más acá), la manifestación directa e inmediata del yo es perfectamente posible. ¿Dónde? Como decíamos antes, en el ámbito de la afectividad, que sigue el esquema de la inmanencia radical y permite la manifestación del sujeto a sí mismo, su auto-sentirse primitivo (Lacroix, 1966, 162 y 163).

Al plantear como nuevo paradigma de acercamiento a la esencia del aparecer el esquema de la afectividad, Henry llega a establecer que el arché buscado por toda filosofía radical y primera no es otra cosa que la Vida misma. Esto es así porque, como nuestro filósofo señala, la Vida es afectividad pura que se manifiesta en cada una de las modalidades de nuestra subjetividad; la materia de la Vida es la pura afectividad o, más bien, la autoafectividad, porque la esencia de la Vida consiste en experimentarse a sí misma, sentirse a sí misma, sufrirse y gozarse a sí misma. Para Henry, el aparecer objetivo en el horizonte de la trascendencia, fuente del conocimiento intramundano propio de las ciencias, solo es posible porque dicho aparecer se recibe a sí mismo en el espacio patético de la Vida; un espacio patético no por ser grotesco o vergonzoso, sino por ser el pathos el modo fenomenológico de revelación propio de la Vida: la "pasión", el "ser afectados" por todo lo que experimentamos y sentimos.

El aparecer que Henry busca en su fenomenología radical-material no es más que la revelación de la realidad en la inmanencia radical y patética de la Vida; una Vida que, en 
su autoafección propia, constituye la ipseidad patética del viviente, estableciéndose, pues, como principio de individuación. Esta ipseidad patética del yo fue caracterizada ya por Maine de Biran (una de las grandes influencias que recibe Michel Henry) como sentimiento de esfuerzo o movimiento; no un movimiento exterior, sino interior, subjetivo, primero y originario que es concebido como poder. En su Fenomenología y filosofía del cuerpo, Henry nos muestra como Maine de Biran reformula la reducción cartesiana al señalar que el pensamiento y la duda también pueden ponerse ellos mismos en cuestión, de forma que la única certeza que nos queda es el ego sentio, el yo siento: no puedo dejar de experimentar, de sentir mi propia capacidad de moverme, mi poder para actuar. Pero esta, desde luego, no es una certeza intelectiva, de conocimiento, sino una certeza que se da como revelación.

Este descubrimiento es, quizá, la fortaleza y radical originalidad, aunque también la parte más controvertida, del pensamiento de Maine de Biran y, por ende, de Michel Henry: la verdadera certeza autoevidente, fundamento del conocimiento y la realidad, no es el pensamiento que se afianza a sí mismo a fuerza de la lógica y la elucidación intelectual, sino algo que aparece como revelación prelógica, algo que siento pero de lo que difícilmente puedo dar cuenta intelectiva, pues en el momento en que me pongo a pensarlo, lo pierdo, me salgo de su inmediata evidencia; un "saber" básico que se produce antes del saber, un "conocimiento" esencial que acontece más acá del conocimiento, un "comprender" fundamental al margen del comprender representativo y que no sólo lo antecede, sino que es su condición de posibilidad. Para mostrarnos esto, Maine de Biran subraya que la mayor parte de nuestra vida se mueve en el terreno del aprendizaje prelógico y prerracional, el aprendizaje cinestésico que nos permite sentir el cuerpo propio y el espacio que ocupamos en el mundo ${ }^{1}$; un sentir que acaba convirtiéndose en un poder, el poder de moverse.

Y este poder, que no es otra cosa que la subjetividad misma, se identifica con el cuerpo que cada uno recibe como "suyo": $m i$ subjetividad no es otra cosa que mi cuerpo. El "cuerpo subjetivo" del que habla Henry por influencia biraniana y al que luego remitirá con otros términos (carne, autoafección, Vida, alma, etc.) aparece, así, como la instancia existencial en la que reposa la "egocidad" del yo, la entidad espiritual-corporal que, aún siendo sentida y recibida como cuerpo propio, difiere del cuerpo material porque padece realmente, porque manifiesta en sí misma cada una de las diferentes modalidades de la afectividad en que consiste la Vida. La experiencia de cualquier afección siempre se produce en este "cuerpo subjetivo", lo que constituye una prueba irrefutable de la inmanencia radical de la conciencia: no existe ninguna afección que no afecte al yo en su inmanencia, ya que si una afección no es advertida por la conciencia ni siquiera mínimamente, simplemente no existe. Por eso Henry se aventura a decir que la afección no necesita referencia alguna a otra cosa que no sea ella misma, es decir, que no es intencional: es el dolor lo que duele, es el gozo lo que regocija².

1 Etimológicamente, cinestesia (o kinestesia) significa "sensación del movimiento" y es la percepción del esquema corporal, de la posición, del equilibrio y, en sentido más fuerte, del espacio y del tiempo, condición de posibilidad de nuestra capacidad de movernos. Hay mecanismos cinestésicos de diferente índole: la sensibilidad interoceptiva (que coordina las sensaciones como el hambre, la sed, el sueño, etc.), la sensibilidad externoceptiva (que articula los datos de los sentidos y procesa el dolor y el placer) y la sensibilidad propioceptiva o postural (que capta los datos necesarios para regular el equilibrio y las acciones necesarias para moverse).

2 Cuando, por ejemplo, nos quemamos una mano, no es el fuego, sino nuestro propio yo (a causa del fuego, eso sí) el que nos hace sufrir; es nuestra propia carne, nuestro propio "cuerpo subjetivo" el que nos hace sufrir y el que, a la vez, está sufriendo. No hay distancia fenomenológica entre lo afectado (la mano quemada) y lo 


\section{La teoría de los tres cuerpos}

Este "cuerpo subjetivo" del que habla Henry para profundizar en su idea de la subjetividad corporal, surge, según Maine de Biran, en un acto originario que corresponde con el sentimiento inmediato e interior del movimiento, un sentimiento que no se acompaña de ningún conocimiento de los medios o instrumentos por los que yo muevo mi "cuerpo objetivo". Dicho "cuerpo objetivo" es mi cuerpo trascendente, la parte física y material de mi ser, tal como se manifiesta a los órganos de los sentidos (por ejemplo, cuando me miro una mano o veo mi reflejo en el espejo) y que puede ser objeto de la ciencia. La diferencia entre el "cuerpo objetivo" y el "cuerpo subjetivo" estriba, pues, en que mientras el primero es un objeto, el segundo es un sentimiento y un poder. Pero este poder encuentra un límite que Henry llama "continuo resistente" y que es el término y, a la vez, el freno que mi capacidad de movimiento y acción encuentra en el mundo. El término "resistente" hace referencia a lo que no se pliega a mi poder, a lo que no sucumbe a mi ego possum, articulando la propia esencia de ese poder; el término "continuo", hace referencia al carácter duradero y estable de esa limitación. ${ }^{3}$

Entre el "cuerpo subjetivo" (el que se revela en la experiencia trascendental del movimiento) y el "cuerpo objetivo/trascendente" (el que es objeto de los sentidos, de la ciencia y, en general, del pensamiento teórico), Maine de Biran y, con él, Henry plantean una tercera dimensión de nuestra existencia subjetivo-corporal: el "cuerpo orgánico", esa región de la realidad que coincide en su ser con el ser del ego, al igual que lo hace el "cuerpo subjetivo", pero que es una extensión interna de este, una parte del mundo que "cede" al movimiento absoluto del "cuerpo subjetivo" y que está dividido en estructuras fijas (a saber, los órganos). De esta forma, entre el "cuerpo subjetivo", que es solamente una fuerza, y el "cuerpo objetivo", que no deja de ser un objeto, aparece el "cuerpo orgánico", una propuesta conceptual que Maine de Biran y Henry utilizan para solventar el problema de la constitución del cuerpo-objeto-del-mundo como cuerpo propio que la subjetividad mueve desde la inmanencia radical y absoluta; es decir, para solucionar el problema del paso del "cuerpo subjetivo" al "cuerpo objetivo". (Henry, 1965).

Para mover el "cuerpo objetivo", la subjetividad "necesita" un elemento intermedio, papel que jugarán estos órganos que el "cuerpo subjetivo" mueve y que constituyen, en conjunto, el "cuerpo orgánico". El "continuo resistente", el mundo del que antes hablábamos es el límite con el que el "cuerpo subjetivo" choca, no solo en un sentido material

afectante (también la mano y no el fuego) o incluso, más allá, ni siquiera es importante la espacialidad de la quemadura: que la quemadura esté en la mano o en otro lugar objetivo no importa, porque donde realmente está la quemadura es en la carne, en el "cuerpo subjetivo".

3 En esta primera etapa de su pensamiento, correspondiente a Filosofía y fenomenología del cuerpo (su primer gran trabajo, a pesar de haberse publicado como segunda tesis), Henry se mantiene aún dentro de la fenomenología tradicional, porque, con este planteamiento, está reconociendo, de alguna forma, el esquema de la intencionalidad: en el aparecer más primitivo, yo encuentro mi subjetividad más absoluta (mi poder de movimiento, mi "cuerpo subjetivo"), pero encuentro también aquello que permanentemente choca con mi poder y lo limita: el mundo; el "continuo resistente" se nos da también como irreductible, porque se funda en la certeza que tenemos de él a partir de nuestra Vida, que es la esfera de la certeza absoluta. Esta idea irá desapareciendo progresivamente, a medida que se vaya desarrollando y perfilando el pensamiento henryano a través de la crítica a la fenomenología intencional; en Encarnación. Una filosofía de la carne, no obstante, volverá a aparecer, aunque completamente reformulada. 
y táctil, sino también en el ámbito de otros sentidos: yo tengo el poder de ver, el poder de oír, pero este poder tiene un límite en el mundo que yo miro u oigo. El "continuo resistente", en esta primera etapa, es el límite de mi subjetividad al vivir; de la misma forma, también mi "cuerpo orgánico" va a aparecer como límite de la fuerza de acción y movimiento: mis propio órganos ponen un límite a mi capacidad de sentir, de actuar, de mover. Lo que ocurre es que, en algún sentido, mi “cuerpo orgánico" cede en esa resistencia y, sin embargo, el mundo es un límite fijo que no cede, contra el que mi poder no puede hacer nada. (Ibíd.).

Aunque hereda y asume este esquema de Maine de Biran, Michel Henry dirige también una importante crítica hacia el mismo, puesto que, según él, Maine de Biran se olvida aquí de la pasividad. Para Maine de Biran, el sentimiento originario es una fuerza, un poder de acción, de movimiento; el yo es poder, acción, esfuerzo. Pero, como hemos señalado más arriba, la originalidad del pensamiento de Henry radica en su referencia a la afectividad y a la pasividad radical de la Vida. La vida no solo transcurre en parámetros de acción, movimiento, esfuerzo, sino que también presenta un elemento muy fuerte de pasividad: lo que yo siento no por mi fuerza de moverme o por mi capacidad de acción, sino simplemente porque lo siento, porque lo sufro en el sentido literal; la Vida que sufre, que padece, que carga. Ese sentimiento de pathos, de pasividad, que antes explicábamos, lleva a Henry a reformular la teoría biraniana de los tres cuerpos mediante el concepto de autoafección. No obstante, hablemos de la subjetividad en términos de poder de movimiento o de pasividad radical, ambas vertientes están haciendo referencia a lo mismo: esa esfera no constituida, que se conoce de forma inmediata e indubitable y que, a diferencia del conocimiento construido sobre la trascendencia exterior del mundo, es el único conocimiento absolutamente certero.

Sea por asunción del esquema biraniano o por su posterior reformulación en términos de absoluta y radical pasividad, encontramos que, para Henry, la existencia humana no es la unión de un "cuerpo trascendente" con un ego, al estilo del dualismo platónico-cristianocartesiano; más bien, la vida del cuerpo es la Vida del ego. No se trata tanto de "tener" un cuerpo, sino de "ser" un cuerpo: "yo soy mi cuerpo". El problema tradicional de la existencia del alma y de su compleja relación con el cuerpo queda resuelto, así, en la fenomenología radical henryana, mediante una concepción del ego como emergencia de una subjetividad corporal de la que el "cuerpo objetivo" no es más que una "representación" en el medio trascendente; un "préstamo" que el mundo recibe del cuerpo subjetivo, una "proyección" del ego en la parte de extensión ocupada en el mundo.

\section{El sufrimiento, la comunidad política, la ética. Implicaciones de la fenomenología henryana}

Esta forma de concebir la subjetividad desde la experiencia corporal nos lleva a plantear algunos fenómenos cotidianos que pueden ser repensados a la luz de la filosofía henryana para motivar la discusión y abrir nuevas vías de investigación; nos centraremos en tres: la experiencia del sufrimiento, la constitución de las comunidades socio-políticas y la emergencia de la dimensión ética. En la primera de estas tres instancias, la clave está en entender que, para Henry, el sufrimiento no se manifiesta originariamente en 
la exterioridad del mundo, sino solo en la inmanencia absoluta de la Vida interior. Si entendemos el sufrimiento en su modo propio de darse, que es la interioridad inmanente del "cuerpo subjetivo", descubrimos que coincide con la condición de posibilidad misma de la Vida, pues la Vida es pura afectividad y la esencia misma de la afectividad es el sufrimiento (ser "afectado" no es sino "sufrir"/"padecer" una afección). El sufrir es, pues, la misma esencia de la Vida (Cazzanelli, 2013, 13 y 14). El Comienzo, el ser mismo, el aparecer del aparecer que busca toda fenomenología comprendida como verdadera "filosofía radical y primera", se manifiesta en el sufrirse-a-si-mismo, que es la esencia de la autoafección. Pero hablar de sufrimiento es también hablar de gozo, porque en el sufrimiento se realiza el ser-dado-a-sí, el disfrute y alegría del ser (Lacroix, $1966,164$ y 165$)$.

En el caso del establecimiento de nuestras comunidades humanas, podemos señalar que la investigación fenomenológica en torno a la afectividad inmanente de la Vida presenta la esencia de la manifestación como aquella instancia que nos capacita para construir verdaderas relaciones interpersonales desde nuestra interioridad personal afectiva (la prueba patética de la Vida en cada singularidad viviente permite el desarrollo de una interioridad fenomenológica recíproca que hace del otro un "sí" co-afectado en su carne por una materia fenomenológica pura en común). La Vida es la condición trascendental de toda comunidad. En lugar de pensar la intersubjetividad según el horizonte intencional de visibilidad, hace falta afirmar que toda comunidad es invisible, al ser el pathos-con (la syn-patheia -la simpatía-, la cum-passio -la compasión-), esto es, la experiencia patética de su propia singularidad, lo que hace de los vivientes una comunidad patética invisible; y el medio en el que se articula esta emergencia de la "vida-en-comunidad" no es sino la Vida misma (Tinland, 2008, 116 y 117). Solo constatando este gesto fundacional previo a toda comunidad efectivamente visible del mundo social podemos articular verdaderos programas políticos de liberación, en tanto vinculados más a un destino común de pulsiones y afectos que a una asociación artificial y deliberada de representaciones y voluntades.

Finalmente, en el caso de la ética, podemos decir que la emergencia de la comunidad de los vivientes engendra un sistema de relaciones que presenta dos elementos constitutivos: la inmanencia y la gratuidad. Puesto que la Vida excluye toda estructura ek-stática en su autodonación, las relaciones que se originan en ella son posibles solo como inmanentes, es decir, fundadas en la copertenencia de los términos de la relación; la Vida constituye, así, la condición de posibilidad del encuentro entre los vivientes. Por su parte, al fundamentarse en la Vida, este sistema de relaciones no se basa en el principio de reciprocidad sino en el de gratuidad, es decir, en la lógica de la donación: en tanto vivientes, la relación de la Vida para con cada ipseidad es la absoluta donación. Es imposible, pues, tocar al otro sin tocar la Vida que es él mismo: ambos, yo y el otro, nos reconocemos constituidos en la Vida, y es este reconocimiento el que funda la autodonación recíproca entre nosotros. El punto de partida para la relación con el otro no es, pues el ego constituyente, sino la Vida en la que todos participamos, condición de posibilidad del encuentro y de la interacción entre los vivientes que nos lleva a respetar al otro siempre en su propia ipseidad patética, fundando la dimensión ética de nuestro habitar en el mundo. (Sánchez Hernández, 2012, 93-95). 
“Yo soy mi cuerpo.” La concepción henryana del ego como emergencia de una subjetividad ... 153

\section{Bibliografía}

Cazzanelli, S. (2013), “Introducción”, en: Henry, M. (2004), La fenomenología radical, la cuestión de Dios y el problema del mal, Madrid, Ediciones Encuentro, 2013.

Domínguez Basalo, A. (1978), "Michel Henry, un filósofo de la inmanencia". Revista Pensamiento, vol. $34, \mathrm{n}^{\circ} 134, \mathrm{pp} .145-176$.

Domingo Moratalla, T. (2006), "Introducción”, en: Henry, M. (1987), La barbarie, Madrid, Caparrós Editores, 2006.

Henry, M. (1963), La esencia de la manifestación, Salamanca, Ediciones Sígueme, 2015.

Henry, M. (1965), Filosofía y fenomenología del cuerpo. Ensayo sobre la ontología de Maine de Biran, Salamanca, Ediciones Sígueme, 2007.

Henry, M. (1985), Genealogía del psicoanálisis, Madrid, Editorial Síntesis, 2002.

Henry, M. (1987), La barbarie, Madrid, Caparrós Editores, 2006.

Henry, M. (1990), Fenomenología material, Madrid, Ediciones Encuentro, 2009.

Lacroix, J. (1966), «Un philosophe du sentiment : Michel Henry ». Panorama de la philosophie française contemporaine. Les Éditions G. Crès et Cie, 1968, pp. 161-167.

Lipsitz, M. (2000), "Michel Henry y la crítica del intuicionismo". A parte rei: revista de filosofía, $\mathrm{n}^{\mathrm{o}} 10$, Octubre 2000.

Tinland, O. (2008), « Auto-affection et individuation en Michel Henry », en: Tinland, O. (directeur), L'individu, Paris, Libraire Philosophique J. VRIN, Collection « Théma », pp. 99-121.

Sánchez Fernández, O. F. (2012), “Autoafección y espiritualidad. Hacia una filosofía de la religión en Michel Henry”, Franciscanum (Universidad de San Buenaventura, Bogotá), vol. LIV, núm. 158, julio-diciembre, pp. 83-97. 
